\title{
Pneumococcal meningitis in adult patients admitted to the Intensive care unit : prognostic factors of clinical outcome
}

Authors: Krastina Jana ${ }^{1}$, Kazune Sigita ${ }^{1,2}$ (Mentor)

${ }^{1}$ Faculty of Medicine, Riga Stradins University, Riga, Latvia

${ }^{2}$ Department of Anesthesiology, Hospital of Traumatology and Orthopaedics, Riga, Latvia

DOI: https://doi.org/10.26800/LV-142-supp5-53

\section{Introduction}

Acute bacterial meningitis is a rapidly progressive bacterial infection of the meninges and subarachnoid space, that can lead to death or long-term disability. Pneumococcal meningitis, caused by Streptococcus pneumoniae remains a potentially devastating disease that carries high mortality and morbidity rates in spite of availability of effective antibiotics, vaccination programmes and skilled acute-care facilities.

\begin{abstract}
Aim
The present study aims at determining factors associated with in-hospital mortality in patients with acute pneumococcal meningitis (APM).

\section{Materials and methods}

We conducted a retrospective cohort study of 23 adult patients consequtively suffering from APM admitted to the Intensive Care Unit (ICU) of Riga East Clinical University hospital in a period from January, 2011 through December, 2018. Clinical features, vital signs at the admission time, cerebrospinal fluid characteristics, venous and arterial blood gas tests, platelet count, the need for mechanical ventilation, number of days spent at hospital and at ICU, start of antibiotic and steroid therapy were recorded. Mental state was assessed using Glasgow Coma Scale (GCS). The severity of the disease within 24 hours of admission to the ICU were detected by Acute Physiology and Chronic Health Evaluation (APACHE II) scale. To determine the extent of an organ function or rate of failure, the sequential organ failure assessment score (SOFA) were used.
\end{abstract}

\section{Results}

Death affected $30.4 \%$ of the series under study $(n=7)$. All patients were admitted directly from emergency department. A total of $52.2 \%(n=12)$ patients required mechanical ventilation. The independent risk factors of patient death were APACHE II score $(p=0.005)$, GCS $(p=0.004)$ and SOFA score (0.017).

\section{Conclusion}

For the prediction of mortality in a patient with APM, all of scoring systems we analyzed had a high accuracy. Current findings suggest that overall severity of the disease may be the major indicator of adverese clinical outcome.

Keywords: Adult; Bacterial meningitis; Intensive care unit; Outcome; Streptococcus pneumoniae 